\title{
Statin Intolerance: Diagnosis and Management
}

\author{
Roopali Khanna ${ }^{1}$ Dandu Himanshu ${ }^{2}$ \\ ${ }^{1}$ Department of Cardiology, Sanjay Gandhi Post Graduate Institute of \\ Medical Sciences, Lucknow, Uttar Pradesh, India \\ 2 Department of Medicine, King George's Medical University, \\ Lucknow, Uttar Pradesh, India \\ Indian J Cardiovasc Dis Women-WINCARS 2017;2:14-20.
}

Address for correspondence Roopali Khanna, MD, DM, Department of Cardiology, Sanjay Gandhi Post Graduate Institute of Medical Sciences, Rae Bareilly Road, Lucknow 226014, Uttar Pradesh, India (e-mail: drroopalik@gmail.com).

\begin{abstract}
Statins are the main cornerstone in the treatment of coronary artery disease. Statins not only reduce the cardiovascular events but also significantly reduce the all-cause mortality. Due to adverse effects of statins, 20 to $30 \%$ of patients discontinue statins without consulting the physician. Most common adverse effects reported are muscle symptoms. Studies have shown that the majority of these patients can tolerate statin

Keywords

- statin intolerance

- statin-induced myopathy

- alternate statin dosing upon rechallenge. Alternative statin dosing, alternate-day statin, or twice-weekly statin dosing are different strategies to be given before changing to nonstatin lipid-lowering therapy. The newer nonstatin lipid-lowering drugs (ezetimibe, PCSK9 inhibitors) can be added if the low-density lipoprotein (LDL) target level can be achieved despite the maximally tolerated statin dose. This article reviews the etiology, clinical symptoms, and management of statin intolerance.
\end{abstract}

\section{Introduction}

Statins are considered to be a cornerstone therapy in patients with coronary artery disease. The efficacy of statins for the prevention of recurrent myocardial infarction (MI) has been demonstrated in several randomized controlled trials. ${ }^{1}$ Largescale analyses of $>170,000$ patients have demonstrated that for each $1.0-\mathrm{mmol} / \mathrm{L}(39-\mathrm{mg} / \mathrm{dL})$ reduction in low-density lipoprotein (LDL) cholesterol, statins reduce the rates of major adverse cardiovascular events (MACEs) by 20 to $25 \%{ }^{2}$ Based on this evidence, guidelines recommend high-intensity statins following acute coronary syndromes. ${ }^{3,4}$ Statins are generally well tolerated, but as many as $20 \%$ of patients report adverse muscle side effects. ${ }^{5,6}$

\section{Effect of Discontinuation of Statins in High-Risk Patients}

Statin intolerance may lead to discontinuation of therapy, which is associated with an increased risk of cardiovascular outcomes. ${ }^{7}$ Serban et al showed discontinuation or downtitration of statin in patients of MI within 1 year increases the risk of recurrent MI by $36 \%$ and coronary heart disease (CHD) events by $46 \%{ }^{8}$ It evaluated 1,741 patients of statin intoler- ance, with 55,567 patients of high statin adherence over a median of 1.9 to 2.3 years of follow-up. The multivariate adjustment showed that hazard ratio (HR) comparing beneficiaries with statin intolerance with those with high statin adherence was 1.50 (95\% confidence interval [CI]: 1.30-1.73) for recurrent MI, 1.51 (95\% CI: 1.34-1.70) for CHD events, and 0.96 (95\% CI: $0.87-1.06$ ) for all-cause mortality (-Fig. 1). Misdiagnosing statin intolerance and its inappropriate discontinuation can lead to increased cardiac events. Therefore, the issue of statin intolerance is of great clinical importance.

\section{How Common Is Statin Intolerance?}

Statin intolerance is defined as inability of a patient to tolerate a statin, either because of the development of a side effect or because of evidence on a blood test elevated markers of liver function or muscle function (creatinine kinase). ${ }^{9}$ The intolerance can be either partial (i.e., only some statins at some doses) or complete (i.e., all statins at any dose). The most common presentation of statin intolerance includes muscle aches, pains, weakness, or cramps, often called myalgias. Muscle symptoms are also known as statin-associated myalgia/ myopathy, statin-induced myalgia/myopathy, or statin-related 


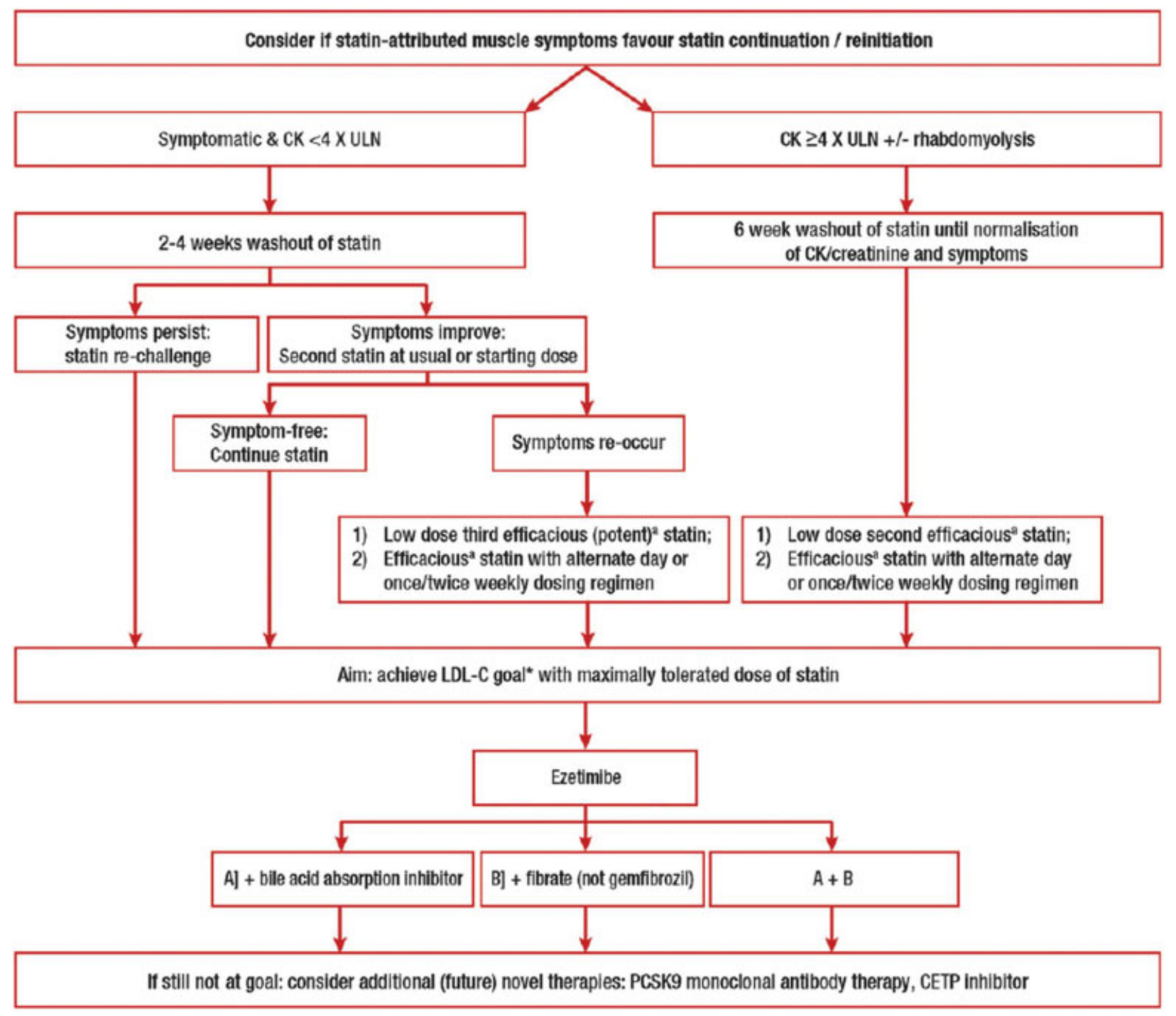

Fig. 1 Flowchart for management of statin-attributed muscle symptoms. (Adapted from EAS Consensus Panel 2015.) CETP, cholesteryl ester transfer protein; CK, creatinine kinase; LDL-C, low-density lipoprotein cholesterol; PCSK9, proprotein convertase subtilisin/kexin type 9; ULN, upper limit of normal range.

myalgia/myopathy or muscle symptoms (SAMS). ${ }^{10}$ The recent statement of the European Atherosclerosis Society (EAS) has focused on SAMS, and avoided the use of the term "statin intolerance," as it is not specific for muscle symptoms. ${ }^{11}$ Although muscle syndromes are the most common adverse effects observed after statin therapy, excluding other side effects might underestimate the number of patients with true statin intolerance. The incidence of statin intolerance in clinical trials varies between 5 and $10 \%$, whereas in observational studies the statin intolerance can be seen up to $20 \% .{ }^{5} \mathrm{~A}$ recent, large, observational survey reports that statin-associated muscle pain affects $29 \%$ of statin users and causes $15 \%$ of these individuals to stop statin therapy. ${ }^{12}$ The reason for lower incidence of statin intolerance in randomized clinical trials (RCTs) can be multifactorial. First, patients with history of any statin related side are excluded in RCTs. Second, patients who are at risk of developing muscle-related symptoms, such as women, elderly patients, and patients with significant comorbidities (e.g., liver failure, kidney failure, or thyroid disease), are underrepresented in RCTs. Definition of statin intolerance is not uniform in trials. Some studies ignored statin-associated myalgia and only considered elevated serum creatine kinase (CK) activity as a side effect. To overcome these issues, International Lipid Panel came with a unified definition of statin intolerance in 2015, which is listed as follows. ${ }^{13}$

1. The inability to tolerate at least two different statins-one statin at the lowest starting average daily dose and the other statin at any dose.

2. Intolerance associated with confirmed, intolerable statin-related adverse effect(s) or significant biomarker abnormalities.

3. Symptoms or biomarker changes resolution or significant improvement upon dose decrease or discontinuation.

4. Symptoms or biomarker changes not attributable to established predispositions such as drug-drug interactions and recognized conditions increasing the risk of statin intolerance. 


\section{Statin Adverse Effects}

- Table 1 summarizes the other side effects mentioned in the literature. Most common adverse effect of statins is muscle related. The symptoms are usually mild and are rarely associated with muscle inflammation (myositis) and markers of muscle injury (creatinine kinase). ${ }^{9}$ These symptoms are completely reversible shortly after the statin discontinuation. Serious muscle damage or rhabdomyolysis associated with statin treatment is extremely rare, for instance, occurring in 1 in 23 million individuals with prescriptions for atorvastatin. Mild to moderate increases in creatinine kinase may be occasionally seen in patients taking statins who have no muscle-related side effects, and this should not be grounds to stop statin therapy.

1. Muscle-related side effects: The most common statinrelated adverse event is muscle weakness; however, due to the many potential reasons for this symptom, it is sometimes difficult to definitely state that it is indeed statin-related. EAS integrated all muscle-related complaints (e.g., pain, weakness, or cramps) as "muscle symptoms," subdivided by the presence or absence of CK elevation (-Table 2). ${ }^{11}$ Pain and weakness in typical SAMS are usually symmetrical and proximal and generally affect large muscle groups, including the thighs, buttocks, calves, and back muscles. Discomfort and weakness typically occur early (within 4-6 weeks after starting statin therapy) but may still occur after many years of treatment. Onset of new symptoms may occur with an increase in statin dose or initiation of an interacting drug. The symptoms appear to be more frequent in physically active individuals. Statin-associated muscle symptoms often appear more promptly when patients are reexposed to the same statin. Commonly SAMS are not accompanied by marked CK elevation. ${ }^{14}$ If CK elevations $>10 \times$ ULN with muscle symptoms it is usually referred to as myopathy, the incidence is approximately 1 per 10,000 per year with a standard statin dose. Rhabdomyolysis is a severe form of muscle damage associated with very high CK levels with myoglobinemia and/or myoglobinuria with a concomitantly increased risk of renal failure. The incidence of rhabdomyolysis in association with statin therapy is 1 in 100,000 per year. ${ }^{15}$ In view of the rarity of CK elevations during statin therapy, routine monitoring of $\mathrm{CK}$ is not recommended. Even if an asymptomatic elevation of CK is detected, the clinical significance is unclear.

\section{Factors Predisposing to Statin-Associated Muscle Symptoms}

-Table 2 summarizes the clinical conditions in which there is increased risk of developing statin-associated muscle symptoms (SAMS). Advanced age, female sex, lower body mass index, and Asian ethnicity predispose to increased risk of adverse effects. Any patient undergoing major surgery predisposes to marked risk of muscle symptoms. The American College of Cardiology (ACC) recommends temporary cessation of statins prior to any major surgery. ${ }^{16}$

2. Hepatotoxicity: An asymptomatic rise in hepatic enzyme activity (elevated aminotransferase) more than three times ULN is seen in $3 \%$ of cases. ${ }^{17}$ The elevation of aminotransferases usually returns to normal after a dose reduction without the need for statin discontinuation. ${ }^{9}$ Statin-induced hepatotoxicity may be present as asymptomatic elevation of serum transaminases ( $0.5-2 \%$ of patients treated), hepatitis,
Table 1 Potential adverse effects of statins

\begin{tabular}{|l|}
\hline Adverse effects with good supportive evidence \\
\hline $\begin{array}{l}\text { Myopathy (muscle aches, cramps, myositis, } \\
\text { rhabdomyolysis) }\end{array}$ \\
\hline Increase in liver function enzymes \\
\hline New-onset diabetes mellitus \\
\hline Adverse effects with little or no supportive evidence \\
\hline Cancer \\
\hline Intracerebral hemorrhage \\
\hline Cognitive decline (Alzheimer's disease) \\
\hline Lung disease \\
\hline Erectile dysfunction \\
\hline Fatigue, headaches, or dizziness \\
\hline Psychiatric illness \\
\hline Cataracts \\
\hline Rheumatoid arthritis \\
\hline Permanent liver or kidney damage \\
\hline Gastrointestinal upset \\
\hline
\end{tabular}

Table 2 Factors predisposing to statin adverse effects

\begin{tabular}{|l|}
\hline High-dose statin therapy \\
\hline Advanced age ( $>70$ y) \\
\hline Female sex \\
\hline Family history of muscle disorders \\
\hline History of creatinine kinase elevation \\
\hline Vitamin D deficiency \\
\hline Renal and hepatic impairment \\
\hline Untreated hypothyroidism \\
\hline Alcohol abuse \\
\hline Asian ethnicity \\
\hline Low body mass index \\
\hline $\begin{array}{l}\text { Genetic polymorphisms (genes associated with drug and } \\
\text { muscle metabolism) }\end{array}$ \\
\hline Heavy or/and unaccustomed exercise \\
\hline Drug abuse (cocaine, amphetamine) \\
\hline Major surgery with metabolic demands \\
\hline
\end{tabular}

Source: Adapted from ESC guidelines for the management of dyslipidemia 2011 
cholestasis, and acute liver failure. Periodic monitoring of liver enzyme activity seems not to be useful in either detecting or preventing serious liver injury. According to the Food and Drug Administration (FDA) recommendation liver function tests are tested at baseline and later as clinically warranted. ${ }^{18}$

3. New-onset diabetes mellitus (NOD): Statin therapy may increase the risk of NOD; this association is observed for all investigated statins (hydrophilic or lipophilic), thus possibly representing a class effect. ${ }^{19}$ For patients on statin therapy, there are no reasons to stop taking statins (even if NOD is diagnosed); however, careful risk stratification before introducing statin therapy should always be performed. Statins should be used in secondary prevention patients, as the cardiovascular benefits significantly outweigh the risk of NOD.

4. Other rare side effects: Despite the fact that SAMS are the most common observed adverse effects, patients on statins may also present with other side effects, which might reduce their quality of life and might require a reduction in dose or statin discontinuation..$^{20}$ - Table 1 summarizes the other side effects mentioned in the literature.

\section{How to Manage Statin Adverse Effects?}

Statin adverse effects depends on the dose of statin, class of statin, age, sex, other comorbidities, and cotreatment with other drugs or foods. Extraneous factors causing rise in CK or liver enzyme levels should be ruled out. Vitamin deficiency, hypothyroidism, and extreme physical exertion can lead to marked increase in CK levels and should be ruled out before discontinuing the therapy.

Patients should be counseled regarding the benefit and harm of statins. Cardiovascular benefits of statins and harms on its discontinuation should be discussed with patients. Reduction in fatal and nonfatal heart attack and stroke by statin treatment cannot be achieved with other currently available medications, nutraceuticals, or dietary modifications. The risk of drug discontinuation versus the benefits must be weighed before making permanent decisions about the management of statin intolerance. The long-term safety and absence of "organ damage" with statins, even when discomfort/pain is present should be reemphasized. There is high likelihood of successful rechallenge with statin and should be explained to the patient. Before considering the use of a second-line alternative drug, patients should try statin rechallenge, alternative regimens, doses, or types of statins. In most cases, rechallenge with a statin after a brief period of drug discontinuation ("drug holiday") can be successful. In a study of 11,124 patients in whom statins were discontinued at least temporarily because of clinical events or symptoms believed to have been caused by statin use, $92 \%$ of those who were rechallenged were still taking a statin 12 months after the statin-related event and $47 \%$ were on same statin to which they had statin-related adverse effects. ${ }^{5}$

\section{Management of Statin-Associated Muscle Symptoms}

Depending on the symptoms and serum CK levels, patients should be stratified ( - Table 3 ). In patients at low cardiovascular disease (CVD) risk with muscle symptoms and CK $<4 \times$ ULN, their need for a statin should be reassessed and the benefits of therapeutic lifestyle changes, such as cessation of cigarette smoking, blood pressure control, and adoption of a Mediterranean style diet, should be balanced against the risk of continuing statin therapy. For patients at high CVD risk, the benefits of ongoing statin therapy need to be weighed against the burden of muscle symptoms. Withdrawal of statin therapy followed by one or more rechallenges (after a washout) can often help in determining causality. Alternative approaches include the use of an alternative statin, a statin at lowest dose, intermittent (i.e., nondaily) dosing of a highly efficacious statin, or the use of other lipid lowering medications. For patients at low CVD risk who have symptoms with $\mathrm{CK}>4 \times \mathrm{ULN}$, the statin should be stopped and its need reassessed. If considered important, a lower dose of an alternative statin should be tried and CK monitored. For patients at high CVD risk with muscle symptoms

Table 3 Definitions of statin-associated muscle symptoms proposed by the EAS Consensus Panel 2015

\begin{tabular}{|l|l|l|}
\hline Symptoms & Biomarker & Comment \\
\hline Muscle symptoms & Normal CK & Often called myalgia; may be related to statin therapy. \\
\hline Muscle symptoms & $\begin{array}{l}\text { CK }<4 \text { ULN } \\
\text { CK }>4<10 \times \text { ULN }\end{array}$ & $\begin{array}{l}\text { Minor elevation of CK with muscle symptoms commonly due to increased exercise } \\
\text { or physical activity; may be statin related; may indicate an increased risk for more } \\
\text { severe underlying muscle problems. }\end{array}$ \\
\hline Muscle symptoms & CK $>10 \times$ ULN & $\begin{array}{l}\text { Called myositis or myopathy; incidence } ~ 1 \text { per 10,000 per year. Pain is typically } \\
\text { generalized and proximal associated with tenderness and weakness. }\end{array}$ \\
\hline Muscle symptoms & CK $>40 \times$ ULN & $\begin{array}{l}\text { Referred to as rhabdomyolysis when associated with renal impairment and/or } \\
\text { myoglobinuria. }\end{array}$ \\
\hline None & CK $<4 \times$ ULN & $\begin{array}{l}\text { Raised CK found incidentally; may be related to statin therapy, rule out thyroid } \\
\text { function or may be exercise related. }\end{array}$ \\
\hline None & CK $<4 \times$ ULN & $\begin{array}{l}\text { Small excess of asymptomatic rises in CK; clinical significance is unclear. Repeat CK } \\
\text { levels if needed. }\end{array}$ \\
\hline
\end{tabular}

Abbreviations: CK, creatinine kinase; ULN, upper limit of normal range.

Source: Adapted from EAS Consensus Panel 2015. 
and a CK of $>4 \times \operatorname{ULN}($ but $<10 \times \mathrm{ULN}$ ), statin therapy can be continued with concomitant monitoring of $\mathrm{CK}$, but it can be stopped (at least temporarily) if the levels exceed $10 \times$ ULN. In this case, that particular statin regimen should not be restarted. If CK levels decrease after stopping the statin, restarting at a lower statin dose with CK monitoring should be tried. In patients with a CK $>10 \times$ ULN, statin therapy should be stopped because of the potential risk of rhabdomyolysis. If the CK level returns to normal, rechallenge with a lower dose of an alternative statin and careful monitoring of symptoms and CK may be considered. If rhabdomyolysis is suspected, statin should not be reintroduced. If there is severe muscular pain, general weakness, and signs of myoglobinemia or myoglobinuria, rhabdomyolysis should be considered. Evaluation of renal damage (urinalysis, serum creatinine levels) should be done. Intravenous hydration and urine alkalinization are recommended for the treatment of rhabdomyolysis depending on severity and the presence of kidney injury. ${ }^{21}$ If indicated, nonstatin low-density lipoprotein cholesterol (LDL-C)-lowering agents should be used (see the following text).

If symptoms/CK abnormalities resolve after discontinuation of statin, either treatment with the same statin at a lower dose or switching to an alternative statin should be started. If tolerated, doses need to be uptitrated to achieve LDL-C goal or as much LDL-C reduction that can be achieved with minimal muscle complaints. If these strategies are not tolerated, alternate-day or twice-weekly dosing can be considered to achieve the LDL-C goal. Studies have shown that either alternate-day or twice-weekly dosing strategies can reduce LDL-C by 12 to $38 \%$, and, importantly, are tolerated by $>70 \%$ of previously intolerant patients. ${ }^{22}$ However, LDL$C$ reduction is much lower than the ideal LDL-C target in alternate dosing, and it has not been established through clinical trials. Thus alternate-day dosing strategy should be used only as a secondary option in specific high-risk patients who are intolerant to lower/alternative statin therapy.

\section{Switching Therapy}

Statins vary in their metabolism, and this affects their potential for drug interaction. Simvastatin and atorvastatin are metabolized via the CYP3A4 pathway. Concurrent therapy with drugs that interfere with CYP3A4 pathway (such as anti retrovirals, cyclosporine, digoxin, diltiazem, and antifungals) may lead to statin accumulation and an increased propensity for myopathic side effects. Statins not metabolized via this pathway such as pravastatin, rosuvastatin, and fluvastatin should be considered in these patients. Similarly, statins that are lipophilic (atorvastatin) have increased incidence of rhabdomyolysis as compared with statins that are hydrophilic (rosuvastatin). Therefore, patients' intolerant to one statin can be switched over to a more hydrophilic statins.

\section{Alternate-Day Dosing}

Statins with longer half-life maintain lipid-lowering effect over a longer period of time, enabling alternate-day dosing strategy with statin. Atorvastatin with a mean half-life of 14 hours is metabolized into two active metabolites: orthohydroxy and parahydroxy forms. Both these active metabolites contribute to $70 \%$ activity of atorvastatin and have a half-life of 20 to 30 hours. This pharmacokinetic parameter of atorvastatin makes it suitable for an alternate-day dosage regimen and continues its lipid-lowering activity for considerably a longer period of time. Rosuvastatin, a third-generation statin, possesses a long half-life period of around 19 hours and can be tried as alternate-dose or twice-weekly dosing.

\section{Nonstatin Lipid-Lowering Therapy}

If LDL-C remains above target despite maximally tolerated statin dosage, addition of an alternative LDL-C-lowering agent should be considered in patients at high CVD risk to improve LDL-C reduction. Ezetimibe decreases the LDL-C level by 15 to $20 \%$ (either in combination with statins or as monotherapy). However, the evidence of cardiovascular benefit is limited to one trial that demonstrated a modest $6 \%$ reduction in cardiovascular events. ${ }^{23}$ Niacin reduces the LDL-C levels by 15 to $18 \%$. However, clinical endpoint trials failed to demonstrate cardiovascular benefits of niacin therapy. ${ }^{24}$ Bile acid sequestrants can reduce LDL-C levels by 15 to $25 \%$ and may also improve glycemia in patients with diabetes. The combination of a bile acid sequestrant and ezetimibe can reduce LDL-C by 30 to $35 \%$. Fibrates are primarily used to lower triglycerides and increase high-density cholesterol; they decrease LDL-C by 10 to $20 \%$. However, additional benefits in CVD with fibrates are not proven, and there is increased risk of raised serum creatinine. ${ }^{25}$ Therefore, we should consider the use of ezetimibe as first choice, potentially followed by bile acid sequestrants or fibrates in combination with ezetimibe, as needed to achieve LDL-C-lowering consistent with guidelines. - Fig. 1 summarizes the approach to patient with SAMS.

\section{Complementary Therapies}

Evidence for the effectiveness of vitamin $\mathrm{D}$ is also controversial, although many patients with SAMS are found to have low blood levels of vitamin D. Hence, routine use of CoQ10 or vitamin $\mathrm{D}$ supplementation is not recommended to treat or prevent SAMS.

\section{Future Low-Density Lipoprotein-Lowering Therapies}

Two classes of novel therapies, proprotein convertase subtilisin/kexin type 9 (PCSK9) inhibitors and cholesteryl ester transfer protein (CETP) inhibitors, has shown to decrease LDL levels similar to statins, and they offer as an alternative to patients with statin intolerance.

\section{Proprotein Convertase Subtilisin/Kexin Type 9 Inhibitors}

Monoclonal antibodies to proprotein convertase subtilisin/ kexin type 9 (PCSK9) have been shown to reduce LDL cholesterol levels in patients who are treated with statins. Studies have shown LDL-C reductions of 50 to $60 \%$ in a patient groups, 
including those identified as statin intolerant, with a very low rate of muscle symptoms, thus reinforcing the concept that statin rather than LDL-C lowering is implicated in the causation of myopathy. ${ }^{26}$ In recent two randomized trials comparing the PCSK9 inhibitor bococizumab with placebo, bococizumab had no benefit with respect to MACEs in the trial involving lower-risk patients but had a significant benefit in the trial involving higher-risk patients (Studies of PCSK9 inhibition and the reduction of vascular events [SPIRE 1 and SPIRE 2 trial]). ${ }^{27}$ Therefore, for high-risk patients with statin intolerance, PCSK9 inhibitors can be used as an alternative.

\section{Cholesteryl Ester Transfer Protease Inhibitor}

Cholesteryl ester transfer protein mediates the heteroexchange of triglycerides and cholesteryl esters between lipoproteins. Inhibitors of CETP markedly increases high-density lipoprotein cholesterol (HDL-C), and two members of this class in advanced development, anacetrapib and evacetrapib, equally lower LDL-C by 25 to $40 \% .{ }^{28}$ Although it decreased LDL-C and increased HDL-C, evacetrapib failed to reduce MACEs when compared with placebo in high vascular risk patients.

\section{Statin Intolerance and Liver Disease}

All patients prior to starting statin should have baseline alanine transaminase/aspartate transaminase (ALT/AST) levels done. Regular monitoring of liver enzymes is not recommended until there is clinical worsening. In patients with chronic liver disease (CLD), guidelines recommend more frequent ALT/AST monitoring; every month during the first 3 months of therapy and four times per year thereafter. In CLD patients, statin therapy should be stopped if AST/ALT activities rise for more than or equal to two times than the baseline value. After AST/ALT activities return to normal/baseline values, it is recommended to consider another statin to establish whether patient is (liver) statin intolerant. With regard to hepatitis B virus (HBV), hepatitis $C$ virus (HCV), and primary biliary cirrhosis (PBC), there are common benefits related to statin therapy in high-risk CV patients. ${ }^{29}$ Statins confer a more efficient treatment of viral hepatitis, and it is related with reduced risk of cirrhosis and hepatocellular carcinoma (HCC). In patients with nonalcoholic fatty liver disease/nonalcoholic steatohepatitis (NAFLD/NASH), statin treatment is safe, contributes to the resolution of NAFLD/NASH, and substantially reduces CVD risk to a level higher than in those with normal liver function.

\section{Conclusion}

Statin intolerance (predominantly muscle symptoms) is a major reason for statin nonadherence/discontinuation; it contributing to decreased CVD benefits from statins. In patients with symptoms of statin intolerance, indication for statin use should be assessed and patients should be made fully aware of the expected benefit in CVD risk reduc- tion that can be achieved with this treatment. Contraindications to use of statin should be ruled out. Patients should be counseled regarding the risk of "side effects" and the high probability that these can be dealt with successfully. Dietary and other lifestyle measures should be emphasized. Statin should be reintroduced after drug washout period. If rechallenge does not work, a low or intermittent dosing should be used preferably of a different (potent or efficacious) statin. Nonstatin therapies should be used as adjuncts as needed to achieve LDL-C goal. Role of supplements is not recommended to alleviate muscle symptoms as there is no good evidence to support their use. Golden principles in management of statin intolerance is always strive to continue "maximally tolerated" statin therapy and always apply repetitive rechallenges. To achieve LDL-C target, different optimal therapy should be combined with maximally tolerated, or even nondaily statin dose, or with nonstatin-based lipid-lowering therapies.

\section{References}

1 Baigent C, Keech A, Kearney PM, et al; Cholesterol Treatment Trialists' (CTT) Collaborators. Efficacy and safety of cholesterollowering treatment: prospective meta-analysis of data from 90,056 participants in 14 randomised trials of statins. Lancet 2005;366(9493):1267-1278

2 Baigent C, Blackwell L, Emberson J, et al; Cholesterol Treatment Trialists' (CTT) Collaboration. Efficacy and safety of more intensive lowering of LDL cholesterol: a meta-analysis of data from 170,000 participants in 26 randomised trials. Lancet 2010;376 (9753):1670-1681

3 Stone NJ, Robinson JG, Lichtenstein AH, et al; American College of Cardiology/American Heart Association Task Force on Practice Guidelines. 2013 ACC/AHA guideline on the treatment of blood cholesterol to reduce atherosclerotic cardiovascular risk in adults: a report of the American College of Cardiology/American Heart Association Task Force on Practice Guidelines. J Am Coll Cardiol 2014;63(25 Pt B):2889-2934

4 Reiner Z, Catapano AL, De Backer G, et al; European Association for Cardiovascular Prevention \& Rehabilitation; ESC Committee for Practice Guidelines (CPG) 2008-2010 and 2010-2012 Committees. ESC/EAS Guidelines for the management of dyslipidaemias: the Task Force for the management of dyslipidaemias of the European Society of Cardiology (ESC) and the European Atherosclerosis Society (EAS). Eur Heart J 2011;32(14):1769-1818

5 Zhang H, Plutzky J, Skentzos S, et al. Discontinuation of statins in routine care settings: a cohort study. Ann Intern Med 2013;158 (07):526-534

6 Mancini GB, Baker S, Bergeron J, et al. Diagnosis, prevention and management of statin adverse effects and intolerance: Canadian Consensus Working Group update (2016). Can J Cardiol 2016;32 (7, Suppl):S35-S65

7 Tong LS, Hu HT, Zhang S, Yan SQ, Lou M. Statin withdrawal beyond acute phase affected outcome of thrombolytic stroke patients: an observational retrospective study. Medicine (Baltimore) 2015;94 (17):e779

8 Serban MC, Colantonio LD, Manthripragada AD, et al. Statin intolerance and risk of coronary heart events and all-cause mortality following myocardial infarction. J Am Coll Cardiol 2017;69(11):1386-1395

9 Mancini GB, Tashakkor AY, Baker S, et al. Diagnosis, prevention, and management of statin adverse effects and intolerance: Canadian Working Group Consensus update. Can J Cardiol 2013;29(12):1553-1568 
10 Thompson PD, Clarkson P, Karas RH. Statin-associated myopathy. JAMA 2003;289(13):1681-1690

11 Stroes ES, Thompson PD, Corsini A, et al; European Atherosclerosis Society Consensus Panel. Statin-associated muscle symptoms: impact on statin therapy-European Atherosclerosis Society Consensus Panel Statement on Assessment, Aetiology and Management. Eur Heart J 2015;36(17):1012-1022

12 Ito MK, Maki KC, Brinton EA, Cohen JD, Jacobson TA. Muscle symptoms in statin users, associations with cytochrome P450, and membrane transporter inhibitor use: a subanalysis of the USAGE study. J Clin Lipidol 2014;8(01):69-76

13 Banach M, Rizzo M, Toth PP, et al. Statin intolerance-an attempt at a unified definition. Position paper from an International Lipid Expert Panel. Arch Med Sci 2015;11(01):1-23

14 Jacobson TA. Toward "pain-free" statin prescribing: clinical algorithm for diagnosis and management of myalgia. Mayo Clin Proc 2008;83(06):687-700

15 Law M, Rudnicka AR. Statin safety: a systematic review. Am J Cardiol 2006;97(8A):52C-60C

16 Fleisher LA, Beckman JA, Brown KA, et al; ACC/AHA TASK FORCE MEMBERS. ACC/AHA 2007 Guidelines on Perioperative Cardiovascular Evaluation and Care for Noncardiac Surgery: Executive Summary: a report of the American College of Cardiology/American Heart Association Task Force on Practice Guidelines (Writing Committee to Revise the 2002 Guidelines on Perioperative Cardiovascular Evaluation for Noncardiac Surgery): Developed in Collaboration with the American Society of Echocardiography, American Society of Nuclear Cardiology, Heart Rhythm Society, Society of Cardiovascular Anesthesiologists, Society for Cardiovascular Angiography and Interventions, Society for Vascular Medicine and Biology, and Society for Vascular Surgery. Circulation 2007;116(17):1971-1996

17 Ginsberg HN, Elam MB, Lovato LC, et al; ACCORD Study Group. Effects of combination lipid therapy in type 2 diabetes mellitus. N Engl J Med 2010;362(17):1563-1574
18 FDA Drug Safety Communication. Important safety label changes to cholesterol-lowering statin drugs (2-28-2012). http://www.fda.gov/ Drugs/DrugSafety/ucm293101.htm. Accessed February 28, 2012

19 Katsiki N, Banach M. Statin use and risk of diabetes mellitus in postmenopausal women. Clin Lipidol 2012;7:267-270

20 Golomb BA, Evans MA. Statin adverse effects : a review of the literature and evidence for a mitochondrial mechanism. Am J Cardiovasc Drugs 2008;8(06):373-418

21 Bosch X, Poch E, Grau JM. Rhabdomyolysis and acute kidney injury. N Engl J Med 2009;361(01):62-72

22 Keating AJ, Campbell KB, Guyton JR. Intermittent nondaily dosing strategies in patients with previous statin-induced myopathy. Ann Pharmacother 2013;47(03):398-404

23 Cannon CP, Blazing MA, Giugliano RP, et al; IMPROVE-IT Investigators. Ezetimibe Added to Statin Therapy after Acute Coronary Syndromes. N Engl J Med 2015;372(25):2387-2397

24 HPS2-THRIVE Collaborative Group; Landray MJ, Haynes R, Hopewell JC, et al. Effects of extended release niacin with laropiprant in high-risk patients. N Engl J Med 2014;371(03):203-12

25 Stein EA. Lipid modulation and atherosclerotic events: extending the clinical spectrum with fibrates and statins. Curr Atheroscler Rep 2000;2(01):11-13

26 Sullivan D, Olsson AG, Scott R, et al. Effect of a monoclonal antibody to PCSK9 on low-density lipoprotein cholesterol levels in statin-intolerant patients: the GAUSS randomized trial. JAMA 2012;308(23):2497-2506

27 Ridker PM, Revkin J, Amarenco P, et al; SPIRE Cardiovascular Outcome Investigators. Cardiovascular efficacy and safety of bococizumab in high-risk patients. N Engl J Med 2017;376(16): 1527-1539

28 Davidson M, Liu SX, Barter P, et al. Measurement of LDL-C after treatment with the CETP inhibitor anacetrapib. J Lipid Res 2013; 54(02):467-472

29 Andrus MR, East J. Use of statins in patients with chronic hepatitis C. South Med J 2010;103(10):1018-1022, quiz 1023 